\title{
Há Necessidade de Solicitar de Rotina Radiografia da Coluna Cervical para Pacientes Portadores de Síndrome de Down Antes de se Realizar Cirurgia Otorrinolaringológica?
}

\author{
There is a Need to Request Cervical Spine Routine Radiographs for \\ Patients with Down's Syndrome Before Carrying out \\ Otorhinolaryngologic Surgery?
}

\author{
Andrea Marçal Szpak*, Bettina Carvalbo**, Juliana Bentbien Cavichiolo**, Marcos Mocellin***, \\ Rodrigo Pereira $* * * *$, Rodrigo Kopp Rezende $* * * * *$.
}

\footnotetext{
* Graduação em Medicina pela Faculdade Evangelica do Paraná. Médico Residente da Disciplina de Otorrinolaringologia do HC/UFPR.

** Graduação em Medicina pela UFPR. Médico Residente da Disciplina de Otorrinolaringologia do HC/UFPR.

*** Doutorado em Otorrinolaringologia pela Escola Paulista de Medicina (1984). Professor Titular da Universidade Federal do Paraná. Presidente da Associação Panamericana de Otorrinolaringologia e Cabeça e Pescoço e Médico Otorrinolaringologista - Clinopar.

***** Otorrinolaringologista pela SBORL. Médico Otorrinolaringologista do Hospital Infantil Pequeno Príncipe.

***** Graduação em Medicina pela Pontifícia Universidade Católica do Paraná. Médico Residente do Serviço de Otorrinolaringologia do HC/UFPR

Instituição: Hospital de Clínicas da Universidade Federal do Paraná.

Curitiba / PR - Brasil.

Endereço para correspondência: Marcos Mocellin - Avenida General Carneiro, 181 - Curitiba / PR - Brasil - CEP: 80060-900 - Telefone: (+55 41) 3360-1800 http://www.hc.ufpr.br/Templates/informacoes/fale/fale.html

Artigo recebido em 13 de Agosto de 2010. Artigo aprovado em 7 de Outubro de 2010.
}

\section{RESUMO}

Introdução:

Objetivo:

Método:

Resultados:

Discussão:

A Síndrome de Down é uma síndrome genética caracterizada por varias alterações, sendo que a Instabilidade Atlanto-axial é de grande importância para o Otorrinolaringologista.

Verificar a prevalência de instabilidade atlanto-axial em pacientes portadores de Síndrome de Down que fazem acompanhamento ambulatorial no Hospital de Clínicas da UFPR e analisar se há necessidade de se realizar de rotina radiografia cervical nos pacientes com indicação de realizar cirurgia otorrinolaringológica.

Estudo prospectivo com paciente portadores da síndrome que tem acompanhamento no HC/UFPR, através de questionário e raios-X cervical.

Não foi encontrado nenhum caso de IAA na população estudada.

Considera-se que há uma alta frequência de IAA em pacientes com Síndrome de Down, sendo recomendado para todos os pacientes que vão participar de atividades esportivas que envolvam movimentação da região ou que sejam submetidos a cirurgias uma investigação com exame clinico e radiológico. No entanto, devido à incidência ter achados muito variados questiona-se a real validade desta investigação para todos os pacientes, mesmo que assintomáticos.

Conclusão:

Apesar de ser uma alteração peculiar da Síndrome de Down, não ha evidencias da necessidade de investigar a IAA rotineiramente em pacientes assintomáticos, sendo que a sintomatologia deve guiar a investigação. Porém, mais estudos são necessários para avaliar a importância dos exames radiológicos nesses casos.

Palavras-chave: síndrome de Down, instabilidade articular, articulação atlanto-axial, radiografia.

\section{SUMMARY}

Introduction:

The Down's Syndrome (DS) is a genetic syndrome characterized by several changes and the Atlanto-axial Instability is critical for the otorhinolaryngologist.

Objective: Check the prevalence of atlanto-axial instability in patients with Down's Syndrome who undergo service followup in the Clinical Hospital of the UFPR [Federal University of Paraná] and review the need to carry out routine cervical radiography in the patients with prescription to otorhinolaryngologic surgery.

Method: Prospective study of patients with the syndrome who undergo CH/UFPR's follow up, through questionnaire and cervical X-ray.

Results:

No case of IAA was found in the population studied. Discussion: we consider there's a high frequency of AAI in patients with DS, and for all patients who will take part in sports activities that involve motion of the region, or who are submitted to surgeries, an investigation with clinical and radiological exam is recommended. However, as the incidence has many variable findings we question the real validity of this research for all patients, even the asymptomatic ones.

Conclusion: In spite of a DS's peculiar change, there are no evidences of the need to research the AAI as a routine in asymptomatic patients and the symptomatology should guide the investigation. But more studies are required to evaluate the importance of the radiological exams in such cases.

Keywords: Down's syndrome, articular instability, atlanto-axial joint, radiography. 


\section{INTRODUÇÃO}

A Síndrome de Down (SD) ou Trissomia do 21 é uma síndrome genética caracterizada por uma anomalia cromossômica (cromossomo 21 extra). Foi descrita inicialmente em 1866 por Sir John L. H. Down, como a primeira anomalia cromossômica detectada na espécie humana, podendo ocorrer de três formas: trissomia livre, translocação ou mosaicismo $(1,2)$

Dentre as várias alterações ortopédicas encontradas nesta síndrome, a Instabilidade Atlanto-axial (IAA) é de grande importância para a Otorrinolaringologia, a qual é representada por uma mobilidade maior que o normal das duas vértebras cervicais superiores, $\mathrm{C} 1$ e C2 (2). Isto ocorre devido à insuficiência do ligamento transverso, principal estabilizador dos movimentos de flexo-extensão entre a primeira e segunda vértebra cervical, e anormalidade da anatomia desta articulação (3).

A incidência de IAA nos portadores de SD varia de 10 a 30\% (3,7), sendo assintomática na maioria destes indivíduos e em apenas 1 a $2 \%$ dos casos em que a IAA é diagnosticada encontram-se sinais e/ou sintomas desta alteração tais como: desconforto cervical, marcha anormal, alteração do controle esfincteriano, lesão do neurônio motor superior, paralisia e morte $(4,7)$. Cerca de $80 \%$ dos pacientes sintomáticos evoluem para instabilidade crônica. A IAA pode ser precedida por trauma cervical, danos causados por esporte, intubação orotraqueal e cirurgia de cabeça e pescoço (5). É necessária avaliação e tratamento com urgência nos sintomáticos e acompanhamento regularmente ao médico associado à prevenção de traumas cervicais para os assintomáticos.

O diagnóstico definitivo de instabilidade da coluna cervical pode ser difícil. A IAA pode ser detectada através da distância entre a face ínfero-posterior do arco anterior de $\mathrm{C} 1 \mathrm{e}$ o processo anterior do odontoide em radiografias em flexão lateral ou extensão, com limite de até $4,5 \mathrm{~mm}$ (8). O paciente com SD que tiver esta medida ultrapassada terá restrições à realização de atividades físicas. No entanto, podem existir problemas no rastreamento radiográfico para IAA, como: técnica radiológica inadequada e falta de acurácia na distância atlanto-axial $(6,7)$.

O objetivo deste trabalho é verificar a prevalência de Instabilidade Atlanto-axial em trinta pacientes portadores de Síndrome de Down que fazem acompanhamento ambulatorial no Hospital de Clínicas da Universidade Federal do Paraná (UFPR) e analisar se há necessidade de se realizar de rotina radiografia cervical nos pacientes com indicação de realizar cirurgia otorrinolaringológica.

\section{MÉTODO}

Realizou-se estudo prospectivo na população de indivíduos portadores de Síndrome de Down em acompanhamento no Hospital de Clínicas da UFPR, na cidade de Curitiba, no ano de 2008.

O estudo teve aprovação do Comitê de Ética em pesquisa do Hospital de Clínicas da UFPR.

Os pais ou responsáveis pelas crianças foram devidamente informados e contactados sobre o objetivo da pesquisa e qual exame seria realizado com finalidade diagnóstica.

Foram selecionados 30 pacientes sendo $13(43,3 \%)$ do sexo masculino e $17(56,7)$ do sexo feminino, cuja idade variou de 2,5 anos a 15 anos, com média de 9 anos.

Após permissão consentida pelos responsáveis foi realizado a aplicação do questionário o qual registrou todo tipo de sintomatologia apresentada pelo paciente: condição geral de saúde, presença ou não de desconforto cervical ou torcicolo, anormalidades da marcha, espasticidade, paralisias, fraqueza de membros, alterações de controle esfincteriano, história prévia de: trauma, intubação orotraqueal, cirurgia cervical, IVAS de repetição, prática de atividade esportiva. Além de detalhes do desenvolvimento motor.

Os pacientes foram então submetidos a exame radiográfico da coluna cervical conforme o protocolo utilizado na disciplina de Radiologia do Hospital de Clínicas e laudado sempre pelo mesmo pesquisador.

O exame complementar utilizado foi radiografia cervical nas posições ântero- posterior, perfil em flexão e extensão em todos os pacientes. Os critérios radiográficos de avaliação da instabilidade foram baseados na medida do intervalo entre a face ínfero-posterior do arco anterior de $\mathrm{C} 1 \mathrm{e}$ o processo anterior do odontoide, Considerou-se normal até 4,5 $\mathrm{mm}$, entre 4,5 a $6 \mathrm{~mm}$ sugestiva de IAA e IAA franca acima de $6 \mathrm{~mm}$ à distância atlanto-odontoidal (Figuras 1 e 2).

\section{RESULTADOS}

Entre os 30 portadores de Síndrome de Down avaliados através de radiografia da coluna cervical perfil em flexão e extensão e ântero-posterior, encontramos todos com a distância entre a face inferoposterior do arco anterior de $\mathrm{C} 1$ e o processo anterior do odontoide menor que $4,5 \mathrm{~mm}$. Dois pacientes apresentaram a distância no limite 


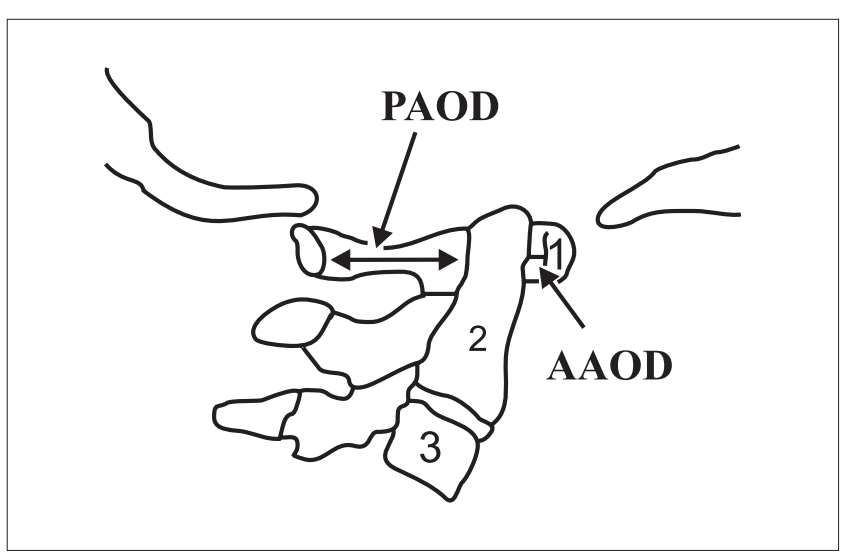

Figura 1. Raio X neutro.

superior da normalidade e encontravam-se assintomáticos. À distância atlanto-odontoidal média na população estudada foi de 3,5 mm e não houve nenhum caso de instabilidade atlanto-axial.

Dentre os 30 pacientes submetidos a questionário sobre sintomatologia, foi constatado que: $2(6,6 \%)$ apresentavam desconforto cervical/torcicolo, 5 (16,6\%) com alterações de controle esfincteriano (maiores de 4 anos de idade) e $1(3,3 \%)$ paciente apresentava queixa de dor em extremidade superior e inferior. Todos tiveram atraso do desenvolvimento motor. Infecção de vias aéreas de repetição foi observada em $50 \%$ dos pacientes.

No entanto, não houve associação significativa entre os sinais e sintomas observados nestes pacientes e as medidas radiológicas.

Os resultados estão resumidos no Gráfico 1.

\section{DISCUSSÃO}

A IAA não traumática foi descrita pela primeira vez por BERKHEISER e SEIDler em 1931; e a alta frequência da subluxação atlanto-axial (SAA) em pacientes com SD por SPITZER em 1961.

Os Membros da Divisão Médica das Olimpíadas Especiais, desde 1983, determinam que todos os participantes portadores de SD devem ser avaliados por exame clínico e radiológico da coluna cervical, a fim de investigar Instabilidade atlanto-axial e se à distância atlanto-odontoidal for maior que $4,5 \mathrm{~mm}$, estes atletas devem ser afastados de atividades esportivas que envolvem movimentos de flexoextensão forçados da coluna cervical para evitar possíveis danos neurológicos devido à compressão medular.

Já a Academia Americana de Pediatria, em 1995,

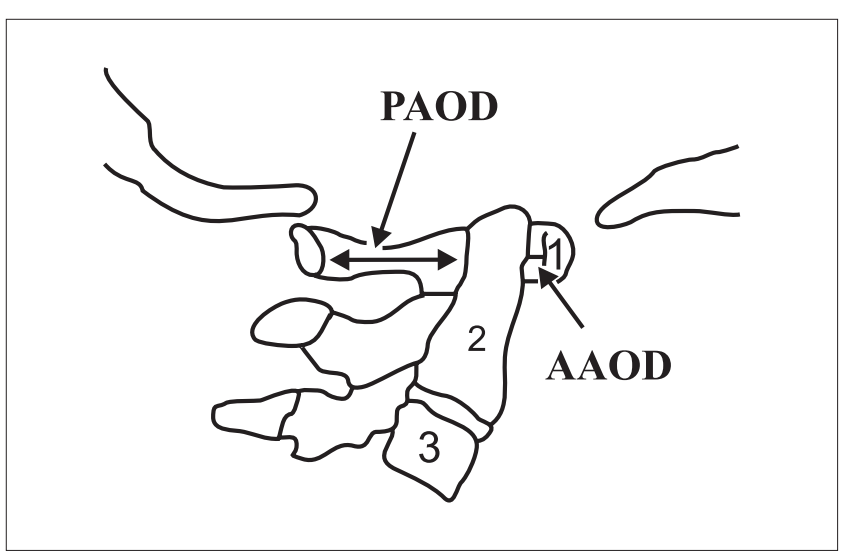

Figura 2. Raio X em flexão.

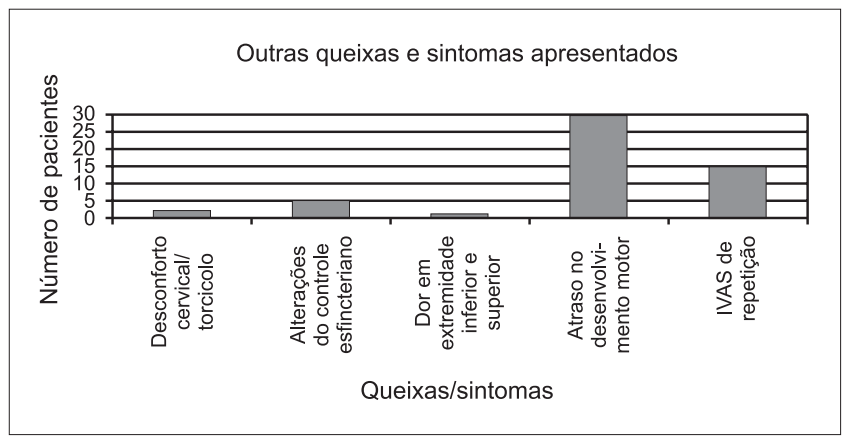

Gráfico 1. Queixas e sintomas apresentados.

questionou a validade da triagem radiológica da IAA em SD, pois, além de ser rara a presença da instabilidade sintomática, geralmente à distância atlanto-axial regride ou estaciona com o passar dos anos.

Mourn et al (9) constatou que, dentre 36 portadores da SD com idade entre 3 e 41 anos ( 16 do sexo masculino e 20 do sexo feminino) apenas 5 casos, ou seja, 13,8\% apresentaram IAA. Outro estudo, realizado por MinATEL et al (10), com 40 portadores de SD, sendo que $15 \%$ foi diagnosticado IAA radiologicamente, porém assintomático enquanto $85 \%$ não apresentaram evidência clinicorradiológica nem limitações quanto à participação em programas de educação física e em atividades esportivas.

Um estudo realizado em Florianópolis por NAHAS et al (1) investigou incidência de IAA em 17 crianças com SD entre 5 e 15 anos, e foi diagnosticado IAA assintomática em 2 delas, ou seja, $11,76 \%$. Durante o trabalho foi constatado em algumas crianças limitação de extensão ou flexão do pescoço, postura cervical apática, hiperextensibilidade cervical e redução da força muscular, não havendo, entretanto, associação nítida entre os sinais observados e as medidas radiológicas. 
Cros, Linares, CaStro e Mansilla (11) realizaram um estudo com 25 portadores de SD, medindo a distância atlanto-odontoidal (DAO) através de radiografias da coluna cervical em flexão e perfil, seguida de tomografia computadorizada e reconstruções tridimensionais. Foi verificado em 4,3\% a DAO maior ou igual a $5 \mathrm{~mm}$, sem variação entre as medidas em flexão e perfil. Através desta pesquisa conclui-se que a incidência da IAA tem achados muito variáveis, de 19 a $30 \%$ de acordo com a literatura. Justifica-se, portanto a necessidade de maiores pesquisas sobre o assunto, visto que neste estudo a incidência da IAA foi 4,3\%. Estes dados estão de acordo com os achados deste estudo, no qual não foi diagnosticado instabilidade atlantoaxial em 30 pacientes portadores de Síndrome de Down submetidos à radiografia da coluna cervical.

Morton et al (12), em acompanhamento de 5 anos, não observou o desenvolvimento de IAA nas crianças estudadas com aferições seriadas.

Em um estudo prospectivo (13), realizado em 1993, foi observado que a prática de esportes não influencia no aparecimento de alterações neurológicas e que o afastamento destes pacientes dos esportes não tem fundamentação científica.

TAYLOR et al (14) concluiu que o diâmetro do canal medular tem maior relevância na avaliação da IAA do que à distância atlanto-axial e quando o canal é menor que 13 mm sugere maior risco de lesão medular. O exame complementar mais adequado para avaliar IAA é a Tomografia Computadorizada e pode ser usada também a Ressonância Magnética. Porém seu uso como screening não é frequente devido à necessidade de realizar indução anestésica e seu alto custo $(15,6)$.

A pesquisa da IAA em pacientes com SD, mesmo assintomáticos, é recomendada quando os mesmos forem submetidos a intubação para anestesia geral ou procedimentos que envolvam hiperflexão ou hiperextensão do pescoço, ou rotação extrema da cabeça (16).

De fato, os pacientes portadores de Síndrome de Down frequentemente necessitam de procedimentos da via aérea superior, como: adenoidectomias e timpanostomias. Durante estes procedimentos é necessário uma posição adequada do pescoço para o cirurgião operar, a qual consiste em extensão importante da coluna cervical para adenoidectomia e rotação de 90 graus para timpanostomia. Conforme a revisão de literatura de Harley e Collins em 1994, é fortemente recomendado que todos os pacientes que forem submetidos a cirurgias otorrinolaringológicas sejam submetidos a um screening pré-operatório para investigar IAA, o que é justificado pelo custo baixo deste screening (17,18). Já CoHEN (19) considera que o screening não é custo-efetivo nem específico, e não é confiável para determinar quais indivíduos com IAA tem risco de desenvolver subluxação ou compressão espinhal.

Não há na literatura um consenso quanto a validade real da triagem radiológica para pesquisar Instabilidade Atlanto-axial em todos os pacientes com Síndrome de Down.

Sugere-se, no entanto que os médicos que realizem procedimentos de risco em pacientes com Síndrome de Down (anestesia, cirurgia com manipulação cervical como otorrinolaringológica) devem tratar todos como susceptíveis, e tomar precauções universais (19).

\section{CONCLUSÃO}

A Instabilidade Atlanto-axial é uma alteração peculiar da Síndrome de Down para a qual não há evidências científicas da necessidade de solicitar de rotina radiológica, tal como preconizado pela Divisão Médica das Olimpíadas Especiais.

Estudos novos devem ser realizados para definir a real importância da pesquisa radiológica da IAA nos pacientes com SD e o controle clínico regular sempre deve ser realizado à procura de a parecimento de alterações neurológicas, que são o principal indício de compressão medular na presença de IAA.

\section{REFERÊNCIAS BIBLIOGRÁFICAS}

1. Nahas MV, Rosário AV, Nahas AB, Luza GR. Instabilidade atlanto-axial em crianças com Síndrome de Down na Grande Florianópolis: um estudo piloto. ACMArq Catarin Méd. 1991, 20(4):149-54.

2. Barros Filho TE, Oliveira RP, Rodrigues NR, Galvão PE, Souza MP. Instabilidade atlanto-axial na Síndrome de Down. Relato de 10 casos tratados cirurgicamente. Rev Bras Ortop. 1998, 33(2):91-4.

3. Siegfried MP. Should Children With Sown Syndrome Be Screened for Atlantoaxial Instability? Arch Pediatr Adolesc Med. 1998, 152:123-125.

4. Ali FE, Al-Bustan AM, Al-Busairi WA, Al-Mulla FA, Esbaita EY. Cervical spine abnormalities associated with Down syndrome. Int Orthopaedics. 2006, 30(4):284-289.

5. Pueschel SM, Scola FH, Tupper TB, PezzulloJC. Skeletal anomalies of the upper cervical spine in children with Down syndrome. J Pediatr Orthop. 1990, 10(5):607-611. 
6. Hata T, Todd, MM. Cervical Spine Considerations when Anesthetizing patients with Down Syndrome. Anesthesiology. 2005, 102(3):680-685.

7. Barros Filho TEP, Oliveira RP, Rodrigues NR, Galvão PE, Souza MP. Instabilidade Atlanto-Axial na Síndrome de Down. Relato de dez casos tratados cirurgicamente. Rev Bras Ortop. 1998, 33(2):91-4.

8. Singer SJ, Rubin IL, Strauss KJ. Atlantoaxial Distance in Patients, Standardization of Measurement. Radiology. 1987, 164:871-872.

9. Molin ED, Molin ECD, Chueire A. Instabilidade de C1-C2 na coluna cervical na Síndrome de Down. Semina. 1988, 9(3):156-8.

10. Minatel E, Ortiz J, Campos RC, Rupp AC. Coluna Cervical na Síndrome de Down. Rev Bras Ortop. 1991, 26(5):1316.

11. Cros'T, Linares R, CastroA, Mansilla F. Estúdio radiológico de lãs alteraciones cervicales em el síndrome de Down. Nuevos hallzgos mediante tomografia computarizada y reconstrucciones tridimensionales. Rev Neurol. 2000, 30(12):1101-7.

12. Morton RE, Khan MA, Murray-LeslieC, ElliottS. Atlantoaxial instability in Down's syndrome: a five year follow up study. Arch Dis Child. 1995, 72(2):115-8; discussion 118-9.

13. Cremers MJ, Bol E, de Roos F, van Gijn J. Risk of sports activities in children with Down's sundrome and atlantoaxial instability. Lancet. 1993, 342(8870):511-4.
14. Taylor TK, Walter WL. Screening of children with Down syndrome for atlantoacial (C1-2) instability: another contentious health question. Med J Aust. 1996, 165(8):44850 .

15. Atlantoaxial instability in Down syndrome: subject review. American Academy of Pediatrics Committee on Sports Medicine and Fitness. Pediatrics. 1995, 96:151-4.

16. Committee on Sports Medicine: Atlantoaxial instability in Down Syndrome. Pediatrics. 1984, 74(1):152-154.

17. Harley EH, Collins MD. Neurologic sequelae secondary to atlantoaxial instability in Down syndrome. Arch Otolaryngol Head Neck Surg 1994; 120:159-165.

18. Brockmeyer D. Down Syndrome and Craniovertebral Instability. Pediatr Neurosurg. 1999, 31:71-77.

19. Cohen WI. Current dilemmas in Down syndrome clinical care: celiac disease, thyroid disorders, and atlanto-axial instability. Am J Med Genet C Semin Med Genet. Aug 15 2006, 142C(3):141-8.

20. Birrer RB. The Special Olympics athlete: evaluation and clearance for participation. Clin Pediatr (Phila). 2004, 43(9):777-82.

21. Pizzutillo PD, Herman MJ. Cervical spine issues in Down syndrome. J Pediatr Orthop. 2005, 25(2):253-9. 